\title{
The Dynamic Similitude Design Method of Thin Walled Structures and Experimental Validation
}

\author{
Zhong Luo, ${ }^{1}$ You Wang, ${ }^{1}$ Yunpeng Zhu, ${ }^{1}$ and Deyou Wang ${ }^{2}$ \\ ${ }^{1}$ School of Mechanical Engineering \& Automation, Northeastern University, Shenyang 110819, China \\ ${ }^{2}$ AVIC Shenyang Aeroengine Design Institute, Shenyang 110042, China \\ Correspondence should be addressed to Zhong Luo; zhluo@mail.neu.edu.cn
}

Received 25 July 2015; Revised 27 September 2015; Accepted 7 October 2015

Academic Editor: Francesco Franco

Copyright (c) 2016 Zhong Luo et al. This is an open access article distributed under the Creative Commons Attribution License, which permits unrestricted use, distribution, and reproduction in any medium, provided the original work is properly cited.

\begin{abstract}
For the applicability of dynamic similitude models of thin walled structures, such as engine blades, turbine discs, and cylindrical shells, the dynamic similitude design of typical thin walled structures is investigated. The governing equation of typical thin walled structures is firstly unified, which guides to establishing dynamic scaling laws of typical thin walled structures. Based on the governing equation, geometrically complete scaling law of the typical thin walled structure is derived. In order to determine accurate distorted scaling laws of typical thin walled structures, three principles are proposed and theoretically proved by combining the sensitivity analysis and governing equation. Taking the thin walled annular plate as an example, geometrically complete and distorted scaling laws can be obtained based on the principles of determining dynamic scaling laws. Furthermore, the previous five orders' accurate distorted scaling laws of thin walled annular plates are presented and numerically validated. Finally, the effectiveness of the similitude design method is validated by experimental annular plates.
\end{abstract}

\section{Introduction}

Thin walled structures are widely used in mechanical and aerospace engineering application due to the excellent dynamic characteristics and high specific stiffness, and it is important to analyze their vibration characteristics $[1,2]$. However, employing the prototype directly in experiments is time consuming and difficult to test. Fortunately, similitude design is a powerful method to predict the dynamic characteristics of the prototype. Due to the limitations of structural parameters of a prototype, for example, the thickness of thin walled structures being difficult to machine in a scaled down model, a distorted model is required to address the problem.

In aspect of the vibration analysis of thin walled structures, Narita [3] analyzed natural characteristics of simply supported plates by using asymptotic expansions and the modified Ritz method. Wang et al. [4] investigated the free vibration characteristics under different boundary conditions based on the differential quadrature method. Irie et al. [5] analyzed the dynamic problems of conic shells with variable thickness, cone-column composite shells, and ring platecolumn composite shells and put forward the transfer matrix method to analyze shells' vibration characteristics under arbitrary boundary conditions. Papkov and Banerjee [6] presented a new method for the free vibration and buckling analysis of rectangular orthotropic plates, which allowed obtaining practically exact results for the free vibration and buckling of orthotropic plates.

The scaling laws of thin walled structures have been investigated by many researchers. Krayterman and Sabnis [7] investigated the scaling laws of a plate by using dimensional analysis method and numerically discussed the accuracy in determining scaling laws. Qian and Swanson [8] obtained the scaling laws of laminated plates with the impulse response, and the scaling laws could accurately describe the undamaged response. Rezaeepazhand and Simitses [9-11] studied the dynamic behaviors of similitude models in predicting the buckling and free vibration of a laminated shell. Wu et al. $[12,13]$ presented scaling laws for the prediction of vibration characteristics using a scaled model by the similitude theory and dimensional analysis. Ungbhakorn and Singhatanadgid [14-16] proposed a new approach of deducing scaling laws for stability and presented scaling laws of symmetrically laminated plates and cylindrical shells by considering the loads 
effects. Oshiro and Alves [17, 18] employed a constitutive law to establish the model and the model reflected exactly as the prototype by changing the impact velocity. Oshiro and Alves [19] investigated the geometrically distorted scaling law in order to predict the behaviors of the prototype, and three problems of the prototype were analyzed. Yazdi [20] obtained the scaling laws that can predict the nonlinear vibration frequency of the prototype, and vibration amplitudes were investigated. De Rosa et al. [21, 22] derived scaling laws for the dynamic response of rectangular flexural plates by the modal approach; the forced response and energy response were given out. In their studies, the structural similitude was investigated by the energy distribution method that allows the representation of all the fundamental parameters. Luo et al. $[23,24]$ investigated the similitude design of an elastic cantilever thin plate by sensitivity analysis, and the distorted scaling laws and size applicable intervals were determined. Further, the prediction of the vibration characteristics of an isotropic sandwich plate was investigated.

In the above studies, the dynamic characteristics and similitude design methods of thin walled plates and cylindrical shells have been investigated by many researchers. However, studies of determining the accurate distorted scaling laws of typical thin walled structures have not been discussed. In this study, the determining method of the accurate distorted scaling laws of thin walled structures is proposed and theoretically proved.

In Section 2 of this paper, the governing equation of typical thin walled structures is firstly unified. Geometrically complete scaling laws are deduced, and three principles of determining accurate distorted scaling law are proposed and proved in Section 3. Furthermore, taking the thin walled annular plate as an example, the distorted scaling laws are obtained in Section 4. Finally, the similitude design method is validated via experimental annular plates.

\section{Governing Equation}

The curved coordinate system $O^{\prime} \alpha \beta \gamma$ is established in the Cartesian coordinated system $O x y z$ and depicted in Figure 1. $\alpha$ and $\beta$ are along with the curvature direction; $\gamma$ is perpendicular to the directions $\alpha$ and $\beta$. There are five internal force components $N_{\alpha}, N_{\beta}, N_{\alpha \beta}, Q_{\alpha}$, and $Q_{\beta}$ and three inner torque components $M_{\alpha}, M_{\beta}$, and $M_{\alpha \beta}$ along with the arc edges of the surface $O \alpha \beta . u, v$, and $w$ represent the tangential displacements of directions $\alpha, \beta$, and $\gamma$, respectively.

The material parameter $E$ is Young's modulus, $\rho$ is density, and $\mu$ is Poisson's ratio. [25]

The arc length $\mathrm{d} r$ of curved surfaces can be denoted as

$$
\mathrm{d} r=(\mathrm{d} s)^{2}=A^{2}(\mathrm{~d} \alpha)^{2}+B^{2}(\mathrm{~d} \beta)^{2}
$$

where $A$ and $B$ are Lamé parameters, $A=$ $\sqrt{(\partial x / \partial \alpha)^{2}+(\partial y / \partial \alpha)^{2}+(\partial z / \partial \alpha)^{2}}$ and $B=$ $\sqrt{(\partial x / \partial \beta)^{2}+(\partial y / \partial \beta)^{2}+(\partial z / \partial \beta)^{2}}$.

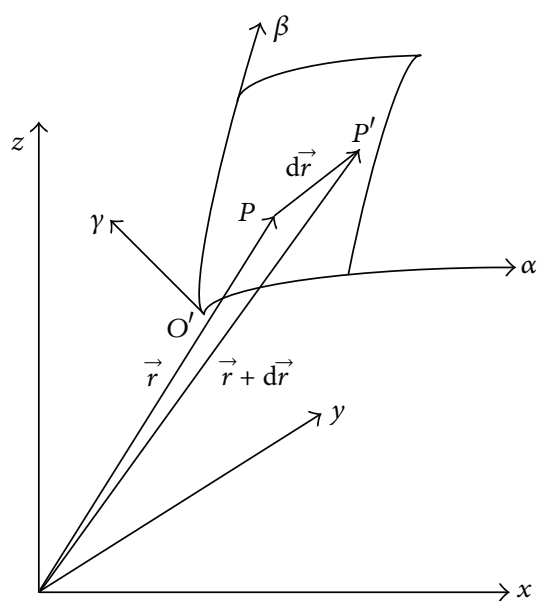

FIgURE 1: The coordinate system.

The internal forces of the infinitesimal surface can be expressed as

$$
\begin{aligned}
N_{\alpha} & =K\left(\varepsilon_{\alpha}^{0}+\mu \varepsilon_{\beta}^{0}\right), \\
N_{\beta} & =K\left(\varepsilon_{\beta}^{0}+\mu \varepsilon_{\alpha}^{0}\right), \\
N_{\alpha \beta} & =N_{\beta \alpha}=K \frac{(1-\mu)}{2} \varepsilon_{\alpha \beta}^{0}, \\
M_{\alpha} & =D\left(\chi_{\alpha}+\mu \chi_{\beta}\right), \\
M_{\beta} & =D\left(\chi_{\beta}+\mu \chi_{\alpha}\right), \\
M_{\alpha \beta} & =M_{\beta \alpha}=D \frac{(1-\mu)}{2} \chi_{\alpha \beta},
\end{aligned}
$$

where $K$ is the membrane stiffness, $K=E h /\left(1-\mu^{2}\right) ; N_{\alpha}$ and $N_{\beta}$ are membrane stresses; $N_{\alpha \beta}$ is the shear stress; $D$ is bending stiffness, $D=E h^{3} / 12\left(1-\mu^{2}\right) ; M_{x}$ and $M_{\theta}$ are the bending moments; $M_{x \theta}$ is the torque; $\varepsilon_{\alpha}^{0}, \varepsilon_{\beta}^{0}$, and $\varepsilon_{\alpha \beta}^{0}$ are strain components, and $\chi_{\alpha}, \chi_{\beta}$, and $\chi_{\alpha \beta}$ are curvature components, which are as follows:

$$
\begin{aligned}
\varepsilon_{\alpha}^{0}= & \frac{1}{A} \frac{\partial u}{\partial \alpha}+\frac{v}{A B} \frac{\partial A}{\partial \beta}+\frac{w}{R_{\alpha}}, \\
\varepsilon_{\beta}^{0}= & \frac{1}{B} \frac{\partial v}{\partial \beta}+\frac{u}{A B} \frac{\partial B}{\partial \alpha}+\frac{w}{R_{\beta}}, \\
\varepsilon_{\alpha \beta}^{0}= & \frac{A}{B} \frac{\partial}{\partial \beta}\left(\frac{u}{A}\right)+\frac{B}{A} \frac{\partial}{\partial \alpha}\left(\frac{v}{B}\right), \\
\chi_{\alpha}= & \frac{1}{A} \frac{\partial}{\partial \alpha}\left(\frac{u}{R_{\alpha}}-\frac{1}{A} \frac{\partial w}{\partial \alpha}\right) \\
& +\frac{1}{A B}\left(\frac{v}{R_{\beta}}-\frac{1}{B} \frac{\partial w}{\partial \beta}\right) \frac{\partial A}{\partial \beta}, \\
\chi_{\beta}= & \frac{1}{B} \frac{\partial}{\partial \beta}\left(\frac{v}{R_{\beta}}-\frac{1}{B} \frac{\partial w}{\partial \beta}\right)+\frac{1}{A B}\left(\frac{u}{R_{\alpha}}-\frac{1}{A} \frac{\partial w}{\partial \alpha}\right) \frac{\partial B}{\partial \alpha},
\end{aligned}
$$




$$
\begin{aligned}
\chi_{\alpha \beta}= & \frac{A}{B} \frac{\partial}{\partial \beta}\left(\frac{u}{A R_{\alpha}}-\frac{1}{A^{2}} \frac{\partial w}{\partial \alpha}\right) \\
& +\frac{B}{A} \frac{\partial}{\partial \alpha}\left(\frac{v}{B R_{\beta}}-\frac{1}{B^{2}} \frac{\partial w}{\partial \beta}\right),
\end{aligned}
$$

where $R_{\alpha}$ and $R_{\beta}$ are main curvature radiuses.

In order to obtain the governing equation, let all the stresses multiply the corresponding arc length and let the inertia force components multiply the infinitesimal area in the directions $\alpha, \beta$, and $\gamma$, respectively, which yields

$$
\begin{aligned}
& \frac{\partial\left(B N_{\alpha}\right)}{\partial \alpha}+\frac{\partial\left(A N_{\beta \alpha}\right)}{\partial \beta}-\frac{\partial B}{\partial \alpha} N_{\beta}+\frac{\partial A}{\partial \beta} N_{\alpha \beta}+A B \frac{Q_{\alpha}}{R_{\alpha}} \\
& -A B \rho h \frac{\partial^{2} u}{\partial t^{2}}=0, \\
& \frac{\partial\left(A N_{\beta}\right)}{\partial \beta}+\frac{\partial\left(B N_{\alpha \beta}\right)}{\partial \alpha}-\frac{\partial A}{\partial \beta} N_{\alpha}+\frac{\partial B}{\partial \alpha} N_{\beta \alpha}+A B \frac{Q_{\beta}}{R_{\beta}} \\
& -A B \rho h \frac{\partial^{2} v}{\partial t^{2}}=0, \\
& \frac{\partial\left(B Q_{\alpha}\right)}{\partial \alpha}+\frac{\partial\left(A Q_{\beta}\right)}{\partial \beta}-A B \frac{N_{\alpha}}{R_{\alpha}}-A B \frac{N_{\beta}}{R_{\beta}} \\
& -A B \rho h \frac{\partial^{2} w}{\partial t^{2}}=0,
\end{aligned}
$$

where the relationships of the shear force $Q_{\alpha}$ and $Q_{\beta}$ can be expressed as

$$
\begin{aligned}
& \frac{1}{A B}\left(\frac{\partial\left(A M_{\beta}\right)}{\partial \beta}+\frac{\partial\left(B M_{\alpha \beta}\right)}{\partial \alpha}-\frac{\partial A}{\partial \beta} M_{\alpha}+\frac{\partial B}{\partial \alpha} M_{\beta \alpha}\right) \\
& \quad=Q_{\beta}, \\
& \frac{1}{A B}\left(\frac{\partial\left(B M_{\alpha}\right)}{\partial \alpha}+\frac{\partial\left(A M_{\beta \alpha}\right)}{\partial \beta}-\frac{\partial B}{\partial \alpha} M_{\beta}+\frac{\partial A}{\partial \beta} M_{\alpha \beta}\right) \\
& \quad=Q_{\alpha}, \quad M_{\alpha \beta}-\frac{M_{\beta \alpha}}{R_{\beta}}=0 . \\
& N_{\alpha \beta}-N_{\beta \alpha}+\frac{R_{\alpha}}{}
\end{aligned}
$$

By using (4) and (5), the governing equation can be written as

$$
\begin{aligned}
& \frac{\partial\left(B N_{\alpha}\right)}{\partial \alpha}+\frac{\partial\left(A N_{\beta \alpha}\right)}{\partial \beta}-\frac{\partial B}{\partial \alpha} N_{\beta}+\frac{\partial A}{\partial \beta} N_{\alpha \beta} \\
& +\frac{1}{R_{\alpha}}\left[\frac{\partial\left(B M_{\alpha}\right)}{\partial \alpha}+\frac{\partial\left(A M_{\beta \alpha}\right)}{\partial \beta}-\frac{\partial B}{\partial \alpha} M_{\beta}+\frac{\partial A}{\partial \beta}\right. \\
& \left.\cdot M_{\alpha \beta}\right]-A B \rho h \frac{\partial^{2} u}{\partial t^{2}}=0,
\end{aligned}
$$

$$
\begin{aligned}
& \frac{\partial\left(A N_{\beta}\right)}{\partial \beta}+\frac{\partial\left(B N_{\alpha \beta}\right)}{\partial \alpha}-\frac{\partial A}{\partial \beta} N_{\alpha}+\frac{\partial B}{\partial \alpha} N_{\beta \alpha} \\
& +\frac{1}{R_{\beta}}\left[\frac{\partial\left(A M_{\beta}\right)}{\partial \beta}+\frac{\partial\left(B M_{\alpha \beta}\right)}{\partial \alpha}-\frac{\partial A}{\partial \beta} M_{\alpha}+\frac{\partial B}{\partial \alpha}\right. \\
& \left.\cdot M_{\beta \alpha}\right]-A B \rho h \frac{\partial^{2} v}{\partial t^{2}}=0, \\
& \frac{\partial}{\partial \alpha}\left\{\frac { 1 } { A } \left[\frac{\partial\left(B M_{\alpha}\right)}{\partial \alpha}+\frac{\partial\left(A M_{\beta \alpha}\right)}{\partial \beta}-\frac{\partial B}{\partial \alpha} M_{\beta}\right.\right. \\
& \left.\left.\quad+\frac{\partial A}{\partial \beta} M_{\alpha \beta}\right]\right\}+\frac{\partial}{\partial \beta}\left\{\frac { 1 } { B } \left[\frac{\partial\left(A M_{\beta}\right)}{\partial \beta}+\frac{\partial\left(B M_{\alpha \beta}\right)}{\partial \alpha}\right.\right. \\
& \left.\left.\quad-\frac{\partial A}{\partial \beta} M_{\alpha}+\frac{\partial B}{\partial \alpha} M_{\beta \alpha}\right]\right\}-A B\left(\frac{N_{\alpha}}{R_{\alpha}}+\frac{N_{\beta}}{R_{\beta}}\right) \\
& \quad-A B \rho h \frac{\partial^{2} w}{\partial t^{2}}=0 .
\end{aligned}
$$

For the typical thin walled structures, Lamé parameter can be written as $A=1$ and $B=R_{0}$. For thin walled cylindrical shells, $R_{0}=R$; for the thin walled plates, $R_{0}=$ $+\infty$. Substituting (2) and (3) into (6a), (6b), and (6c), the governing equation of thin walled structures can be denoted as

$$
\begin{aligned}
- & \frac{B D}{A^{2} R_{\alpha}} \frac{\partial^{3} w}{\partial \alpha^{3}}-\frac{D}{B R_{\alpha}} \frac{\partial^{3} w}{\partial \alpha \partial \beta^{2}}+\frac{B}{A}\left(K+\frac{D}{R_{\alpha}^{2}}\right) \frac{\partial^{2} u}{\partial \alpha^{2}} \\
& +\frac{A(1-\mu)}{2 B}\left(K+\frac{D}{R_{\alpha}^{2}}\right) \frac{\partial^{2} u}{\partial \beta^{2}} \\
& +(1+\mu)\left(\frac{K}{2}+\frac{D}{2 R_{\alpha} R_{\beta}}\right) \frac{\partial^{2} v}{\partial \alpha \partial \beta} \\
& +B K\left(\frac{1}{R_{\alpha}}+\frac{\mu}{R_{\beta}}\right) \frac{\partial w}{\partial \alpha}-A B \rho h \frac{\partial^{2} u}{\partial t^{2}}=0 \\
- & \frac{D}{A R_{\beta}} \frac{\partial^{3} w}{\partial \alpha^{2} \partial \beta}-\frac{A D}{B^{2} R_{\beta}} \frac{\partial^{3} w}{\partial \beta^{3}} \\
& +\frac{(1+\mu)}{2}\left(K+\frac{D}{R_{\alpha} R_{\beta}}\right) \frac{\partial^{2} u}{\partial \alpha \partial \beta} \\
& +\frac{B(1-\mu)}{2 A}\left(K+\frac{D}{2 R_{\beta}^{2}}\right) \frac{\partial^{2} v}{\partial \alpha^{2}} \\
& +\frac{A}{B}\left(K+\frac{D}{R_{\beta}^{2}}\right) \frac{\partial^{2} v}{\partial \beta^{2}}+A K\left(\frac{\mu}{R_{\alpha}}+\frac{1}{R_{\beta}}\right) \frac{\partial w}{\partial \beta} \\
& \frac{B t^{2}}{2}(K)
\end{aligned}
$$




$$
\begin{aligned}
- & \frac{B D}{A^{3}} \frac{\partial^{4} w}{\partial \alpha^{4}}-\frac{2 \mu D}{A B} \frac{\partial^{4} w}{\partial \alpha^{2} \partial \beta^{2}}-\frac{2 D(1-\mu)}{A B} \frac{\partial^{4} w}{\partial \alpha^{2} \partial \beta^{2}} \\
- & \frac{A D}{B^{3}} \frac{\partial^{4} w}{\partial \beta^{4}}+\frac{B D}{A^{2} R_{\alpha}} \frac{\partial^{3} u}{\partial \alpha^{3}}+\frac{D}{B R_{\alpha}} \frac{\partial^{3} u}{\partial \alpha \partial \beta^{2}} \\
& +\frac{D}{A R_{\beta}} \frac{\partial^{3} v}{\partial \alpha^{2} \partial \beta}+\frac{A D}{B^{2} R_{\beta}} \frac{\partial^{3} v}{\partial \beta^{3}}-\left(\frac{B K}{R_{\alpha}}+\frac{\mu B K}{R_{\beta}}\right) \frac{\partial u}{\partial \alpha} \\
& +\left(\frac{\mu A K}{R_{\alpha}}+\frac{A K}{R_{\beta}}\right) \frac{\partial v}{\partial \beta} \\
& +\left(\frac{A B K}{R_{\alpha}^{2}}+\frac{2 \mu A B K}{R_{\alpha} R_{\beta}}+\frac{A B K}{R_{\beta}^{2}}\right) w \\
& -A B \rho h \frac{\partial^{2} w}{\partial t^{2}}=0 .
\end{aligned}
$$

From (7a), (7b), and (7c), we can know that the highest order's derivation of the displacements $u, v$, and $w$ with $\alpha$ and $\beta$ is 4 . Therefore, the governing equation of typical thin walled structures can be summarized as

$$
\sum_{j=u, v, w} \sum_{k=1}^{m=4} \sum_{i=0}^{n=k} L_{j-k i} \frac{\partial^{k} j}{\partial \alpha^{k-i} \partial \beta^{i}}+\sum_{j=u, v, w} L_{j} \frac{\partial^{2} j}{\partial t^{2}}=0
$$

where $j$ represents the displacements $u, v$, and $w ; L_{j-k i}$ and $L_{j}$ are corresponding coefficients; $t$ is time.

\section{Derivation of Scaling Laws}

3.1. Geometrically Complete Scaling Law. The governing equation of the prototype and model can be written as

$$
\begin{gathered}
\sum_{j=u, v, w} \sum_{k=1}^{m=4} \sum_{i=0}^{n=k} L_{j \mathrm{p}-k i} \frac{\partial^{k} j_{\mathrm{p}}}{\partial \alpha_{\mathrm{p}}^{k-i} \partial \beta_{\mathrm{p}}^{i}}=\sum_{j=u, v, w} L_{j \mathrm{p}} \frac{\partial^{2} j_{\mathrm{p}}}{\partial t^{2}}, \\
\sum_{j=u, v, w} \sum_{k=1}^{m=4} \sum_{i=0}^{n=k} L_{j \mathrm{~m}-k i} \frac{\partial^{k} j_{\mathrm{m}}}{\partial \alpha_{\mathrm{m}}^{k-i} \partial \beta_{\mathrm{m}}^{i}}=\sum_{j=u, v, w} L_{j \mathrm{~m}} \frac{\partial^{2} j_{\mathrm{m}}}{\partial t^{2}},
\end{gathered}
$$

where subscript $\mathrm{p}$ denotes the prototype and subscript $\mathrm{m}$ denotes the model.

The deflection equation can be denoted as

$$
j(\alpha, \beta, t)=J(\alpha, \beta) e^{i \omega t},
$$

where $\omega$ is the natural frequency; $J$ represents mode functions $U, V$, and $W$.

Substituting scaling factors $\lambda_{e}=\left(e_{\mathrm{m}} / e_{\mathrm{p}}\right)\left(e=j, L_{j-k i}, \alpha\right.$, $\beta, L_{j}, J$, etc.) into (9a) yields

$$
\begin{gathered}
\sum_{j=u, v, w} \sum_{k=1}^{4} \sum_{i=0}^{k} \lambda_{L_{j-k i}} \frac{\lambda_{J}}{\lambda_{\alpha}^{k-i} \lambda_{\beta}^{i}} L_{j \mathrm{~m}-k i} \frac{\partial^{k} J_{\mathrm{m}}}{\partial \alpha_{\mathrm{m}}^{k-i} \partial \beta_{\mathrm{m}}^{i}} \\
=\sum_{j=u, v, w} \lambda_{L_{j}} \lambda_{J} \lambda_{\omega}^{2} L_{j \mathrm{~m}} \frac{\partial^{2} J_{\mathrm{m}}}{\partial t^{2}} .
\end{gathered}
$$

According to the similitude theory, the corresponding coefficient of a prototype's governing equation is proportional to the coefficient of a model, which means

$$
\lambda_{L_{j-k i}} \frac{\lambda_{J}}{\lambda_{\alpha}^{k-i} \lambda_{\beta}^{i}}=\lambda_{L_{j}} \lambda_{J} \lambda_{\omega}^{2}
$$

Under the condition of geometrically complete similitude, this yields

$$
\begin{aligned}
\lambda_{\alpha} & =\lambda_{R_{\beta}} \lambda_{\beta}=\lambda_{h}=\lambda, \\
\lambda_{\mu} & =1, \\
\frac{\lambda_{L_{j, k i}}}{\lambda_{L_{j}}} & =\frac{\lambda_{D}}{\lambda_{\rho} \lambda_{h}}=\frac{\lambda_{K}}{\lambda_{\rho} \lambda_{h}} \lambda^{2}=\frac{\lambda_{E}}{\lambda_{\rho}} \lambda^{2}, \\
\lambda_{\alpha}^{k-i} \lambda_{\beta}^{i} & =\lambda^{4} .
\end{aligned}
$$

Substituting (13) into (12) yields

$$
\lambda_{\omega}=\sqrt{\frac{\lambda_{L_{j-k i}}}{\lambda_{L_{j}}} \frac{1}{\lambda_{\alpha}^{k-i} \lambda_{\beta}^{i}}}=\sqrt{\frac{\lambda_{E}}{\lambda_{\rho}} \lambda^{2} \frac{1}{\lambda^{4}}}=\frac{1}{\lambda} \sqrt{\frac{\lambda_{E}}{\lambda_{\rho}}} .
$$

Therefore, geometrically complete scaling law can be denoted as

$$
\lambda_{\omega}=\frac{1}{\lambda} \sqrt{\frac{\lambda_{E}}{\lambda_{\rho}}}
$$

3.2. Distorted Scaling Law. Normally, there will be many limitations in employing the geometrically complete similitude model in experiments, so it is necessary that designing geometrically distorted models predicts dynamic characteristics of the prototype. Geometrically distorted models are defined such that scaling factors of geometrical parameters of models are keeping different [26].

In addition, (12) can be written as

$$
\lambda_{\omega}=\sqrt{\frac{\lambda_{L_{j-k i}}}{\lambda_{L_{j}} \lambda_{\alpha}^{k-i} \lambda_{\beta}^{i}}}
$$

Therefore, there are many possible candidate distorted scaling laws as $i=0,1, \ldots, 4$. According to (7a)-(7c), scaling factors $\lambda_{L_{j-k i}}=\lambda_{E}^{m} \lambda_{\alpha}^{o^{\prime}} \lambda_{\beta}^{s^{\prime}} \lambda_{h}^{g}$ and $\lambda_{L_{j}}=\lambda_{\rho}^{-n} \lambda_{h}^{g^{\prime}}$ are obtained, and the distorted scaling law can be denoted as

$$
\lambda_{\omega}=\sqrt{\lambda_{E}^{m} \lambda_{\rho}^{n} \lambda_{\alpha}^{o} \lambda_{\beta}^{s} \lambda_{h}^{q}}
$$

In general, the indexes $m$ and $n$ are explicit and can be directly determined according to the governing equation. However, indexes $o, s$, and $q$ may be implicit in the governing equation $[23,24]$. For example, for the distorted scaling law of the thin walled annular plates, the scaling factors of outer and inner radiuses are hiding in the radius scaling factor $\lambda_{r}$ in (31). In the investigation, a new method is proposed to determine the accurate distorted scaling law by combining the governing equation and the sensitivity analysis. 
3.3. The Principles for Dynamic Scaling Laws. The sensitivity is the change rate of structural characteristic parameters with respect to structural parameters $[27,28]$. Three basic principles are proposed and theoretically proved in order to determine the accurate distorted scaling law based on the sensitivity analysis.

Principle 1. In distorted scaling laws, if the scaling factor $\lambda_{j}$ is explicit and presented in the governing equation, the index of scaling factor $\lambda_{j}$ can be directly determined.

Proof. From (7a), (7b), and (7c), scaling factors of parameters $E$ and $\rho$ are explicit and reflected in the governing equation, so scaling factors' indexes of the parameters $E$ and $\rho$ are directly determined. For example, in the numerical validation, parameters $E$ and $\rho$ are directly presented in (29). Therefore, the scaling factors $\lambda_{E}$ and $\lambda_{\rho}$ can be directly determined. However, in the governing equation (29), the scaling factors $\lambda_{a}$ and $\lambda_{b}$ are coupled in the scaling factor $\lambda_{r}$ and the scaling factors $\lambda_{a}$ and $\lambda_{b}$ could not be directly determined and need to employ the following principles.

Principle 2. When sensitivity's absolute values satisfy $\left|\Phi_{1}\right|>$ $\left|\Phi_{2}\right|>\cdots>\left|\Phi_{n}\right|$, the index relation of the scaling factor $\lambda_{n}^{k_{n}}$ is $\left|k_{1}\right|>\left|k_{2}\right|>\cdots>\left|k_{n}\right|$.

$\Phi_{n}$ is the sensitivity of the natural frequency with respect to the structural parameter $n ; k_{n}$ is the index of the scaling factor $\lambda_{n}^{k_{n}}$.

Proof. The sensitivity is applied to the condition of small variations of structural parameters, but the distorted models did not satisfy the condition. So the transitional model is introduced; let

$$
\lambda_{j}=\frac{j_{\mathrm{t}}}{j_{\mathrm{p}}} \frac{j_{\mathrm{m}}}{j_{\mathrm{t}}}=\lambda_{j, \mathrm{p}-\mathrm{t}} \lambda_{j, \mathrm{t}-\mathrm{m}},
$$

where subscript $\mathrm{t}$ denotes the transitional model; a transitional model can be defined as one structural parameter of the prototype changes in a small range, and other parameters remain unchanged; $\lambda_{j, \mathrm{p}-\mathrm{t}}$ is the scaling factor between the prototype and transitional model; $\lambda_{j, \mathrm{t}-\mathrm{m}}$ is the scaling factor between the transitional model and distorted model.

Equation (17) can be written as

$$
\lambda_{\omega}=\sqrt{\lambda_{E}^{A^{\prime}} \lambda_{\rho}^{B^{\prime}} \lambda_{\alpha, \mathrm{p}-\mathrm{t}}^{o} \lambda_{\beta, \mathrm{p}-\mathrm{t}}^{s} \lambda_{h, \mathrm{p}-\mathrm{t}}^{q} \lambda_{\alpha, \mathrm{t}-\mathrm{m}}^{o} \lambda_{\beta, \mathrm{t}-\mathrm{m}}^{s} \lambda_{h, \mathrm{t}-\mathrm{m}}^{q}}
$$

According to the geometrically complete scaling law, the transitional model and distorted model should satisfy $\lambda_{\alpha, \mathrm{t}-\mathrm{m}}=\lambda_{\beta, \mathrm{t}-\mathrm{m}}=\lambda_{h, \mathrm{t}-\mathrm{m}}=\lambda$. Therefore, (19) can be expressed as

$$
\begin{aligned}
\lambda_{\omega} & =\sqrt{\lambda_{E}^{A^{\prime}} \lambda_{\rho}^{B^{\prime}} \lambda_{\alpha, \mathrm{p}-\mathrm{t}}^{o} \lambda_{\beta, \mathrm{p}-\mathrm{t}}^{s} \lambda_{h, \mathrm{p}-\mathrm{t}}^{q} \lambda_{\alpha, \mathrm{t}-\mathrm{m}}^{o} \lambda_{\beta, \mathrm{t}-\mathrm{m}}^{s} \lambda_{h, \mathrm{t}-\mathrm{m}}^{q}} \\
& =\sqrt{\lambda_{E}^{A^{\prime}} \lambda_{\rho}^{B^{\prime}} \lambda_{\alpha, \mathrm{p}-\mathrm{t}}^{o} \lambda_{\beta, \mathrm{p}-\mathrm{t}}^{s} \lambda_{h, \mathrm{p}-\mathrm{t}}^{q} \lambda^{o+s+q}} .
\end{aligned}
$$

If $\lambda_{j \mathrm{t}} \rightarrow \lambda_{j \mathrm{p}}=1$, the sensitivity of a prototype can be denoted as

$$
\Phi_{j \mathrm{p}}=\frac{d \omega_{j}}{d \lambda_{j}}=\frac{\omega_{\mathrm{p}}-\omega_{j \mathrm{t}}}{\lambda_{\mathrm{p}}-\lambda_{j \mathrm{t}}}=\frac{\omega_{\mathrm{p}}-\omega_{j \mathrm{t}}}{1-\lambda_{j \mathrm{t}}},
$$

where $\Phi_{j \mathrm{p}}$ is the sensitivity of a prototype; $\omega_{j \mathrm{t}}$ denotes natural frequency of the transitional model.

If $\left|\Phi_{\alpha \mathrm{p}}=\left(\omega_{\mathrm{p}}-\omega_{\alpha \mathrm{t}}\right) /\left(1-\lambda_{\alpha \mathrm{t}}\right)\right|>\mid \Phi_{\beta \mathrm{p}}=\left(\omega_{\mathrm{p}}-\omega_{\beta \mathrm{t}}\right) /(1-$ $\left.\lambda_{\beta \mathrm{t}}\right) \mid$ is satisfied, under the condition of $\lambda_{\alpha \mathrm{t}}=\lambda_{\beta \mathrm{t}}, \mid \lambda_{\alpha \omega}=$ $\omega_{\alpha \mathrm{t}} / \omega_{\mathrm{p}}|>| \lambda_{\beta \omega}=\omega_{\beta \mathrm{t}} / \omega_{\mathrm{p}} \mid$ can be obtained from (21).

Therefore, $\left|\lambda_{\alpha \omega}\right|>\left|\lambda_{\beta \omega}\right|$ can be obtained under the condition of $\lambda_{\alpha \mathrm{t}}=\lambda_{\beta \mathrm{t}}$.

By combining (19), the relation $o>s$ of scaling factors $\lambda_{\alpha, \mathrm{p}-\mathrm{t}}^{o}$ and $\lambda_{\beta, \mathrm{p} \text { - }}^{s}$ can be deduced if $\left|\Phi_{\alpha \mathrm{p}}\right|>\left|\Phi_{\beta \mathrm{p}}\right|$ is satisfied.

According to the recursive relation, if $\left|\Phi_{1}\right|>\left|\Phi_{2}\right|>$ $\ldots>\left|\Phi_{n}\right|$ is satisfied when there are many parameters, the index relation of scaling factors $\lambda_{n}^{k_{n}}$ should be satisfied as $\left|k_{1}\right|>\left|k_{2}\right|>\cdots>\left|k_{n}\right|$, so Principle 2 is proved.

Principle 3 . If the sensitivity $\Phi_{j \mathrm{p}}>0, \lambda_{j}$ is positively proportional to $\lambda_{\omega}$ in the distorted scaling law; conversely, $\lambda_{j}$ is inversely proportional to $\lambda_{\omega}$ if the sensitivity $\Phi_{j \mathrm{p}}<0$.

Proof. If the sensitivity $\Phi_{j \mathrm{p}}>0$, two cases are as follows.

(1) If the scaling factor $\lambda_{j}$ is satisfied, $\lambda_{j, \mathrm{p}-\mathrm{t}}=j_{\mathrm{t}} / j_{\mathrm{p}}<$ $\lambda_{j \mathrm{p}}=1$, then the natural frequency is satisfied as $\omega_{j, \mathrm{p}-\mathrm{t}}<\omega_{\mathrm{p}}$ according to (21). Further, $\lambda_{\omega}=\omega_{j, \mathrm{p}-\mathrm{t}} / \omega_{\mathrm{p}}<1$ is obtained. Therefore, the scaling factor $\lambda_{\omega}$ will reduce if the scaling factor $\lambda_{j}$ decreases. That is, $\lambda_{j}$ is positively proportional to $\lambda_{\omega}$ in the distorted scaling law.

(2) If $\lambda_{j, \mathrm{p}-\mathrm{t}}=j_{\mathrm{t}} / j_{\mathrm{p}}>\lambda_{j \mathrm{p}}=1$, then $\omega_{j, \mathrm{p}-\mathrm{t}}>\omega_{\mathrm{p}}$. As a result of case (1), $\lambda_{j}$ is positively proportional to $\lambda_{\omega}$ in the distorted scaling law.

When the sensitivity $\Phi_{j \mathrm{p}}<0$, there are similar cases and Principle 3 is proved in the same way.

In addition, the above three principles could obtain the approximate distorted scaling laws. In order to determine the accurate distorted scaling laws, an additional principle is required.

Additional Principle. In the distorted scaling law, the index ratio $k_{1}: k_{2}: \cdots: k_{n}$ of the scaling factor $\lambda_{n}^{k_{n}}$ is approximate to the ratio of the sensitivity values

$$
k_{1}: k_{2}: \cdots: k_{n} \approx \Phi_{1}: \Phi_{2}: \cdots: \Phi_{n} .
$$

Proof. When the structural parameters $\alpha$ and $\beta$ change within the limit range $\left(\lambda_{\alpha \mathrm{t}}, \lambda_{\beta \mathrm{t}} \in[1-\varepsilon, 1+\varepsilon] ; \varepsilon\right.$ is indefinitely small), the distorted scaling laws (20) can be written as

$$
\begin{aligned}
& \lambda_{\alpha \omega, \mathrm{p}-\mathrm{t}}=\frac{\omega_{\alpha, \mathrm{p}-\mathrm{t}}}{\omega_{\mathrm{p}}}=\lambda_{\alpha, \mathrm{p}-\mathrm{t}}^{o / 2}, \\
& \lambda_{\beta \omega, \mathrm{p}-\mathrm{t}}=\frac{\omega_{\beta, \mathrm{p}-\mathrm{t}}}{\omega_{\mathrm{p}}}=\lambda_{\beta, \mathrm{p}-\mathrm{t}}^{s / 2}
\end{aligned}
$$

The sensitivity of natural frequency with respect to parameters $\alpha$ and $\beta$ can be expressed as

$$
\begin{aligned}
& \Phi_{\alpha}=\frac{\mathrm{d} \omega}{\mathrm{d} \lambda_{\alpha}}=\frac{\Delta \omega}{\Delta \lambda_{\alpha}}=\frac{\omega_{\mathrm{p}}-\omega_{\alpha, \mathrm{p}-\mathrm{t}}}{1-\lambda_{\alpha, \mathrm{p}-\mathrm{t}}}, \\
& \Phi_{\beta}=\frac{\mathrm{d} \omega}{\mathrm{d} \lambda_{\beta}}=\frac{\Delta \omega}{\Delta \lambda_{\beta}}=\frac{\omega_{\mathrm{p}}-\omega_{\beta, \mathrm{p}-\mathrm{t}}}{1-\lambda_{\beta, \mathrm{p}-\mathrm{t}}} .
\end{aligned}
$$


Therefore, this yields

$$
\begin{aligned}
\frac{\Phi_{\alpha}}{\Phi_{\beta}} & =\frac{\omega_{\mathrm{p}}-\omega_{\alpha \mathrm{t}}}{1-\lambda_{\alpha \mathrm{t}}} \frac{1-\lambda_{\beta, \mathrm{p}-\mathrm{t}}}{\omega_{\mathrm{p}}-\omega_{\beta, \mathrm{p}-\mathrm{t}}} \\
& =\frac{\omega_{\alpha, \mathrm{p}-\mathrm{t}}\left(\omega_{\mathrm{p}} / \omega_{\alpha, \mathrm{p}-\mathrm{t}}-1\right)}{1-\lambda_{\alpha, \mathrm{p}-\mathrm{t}}} \frac{1-\lambda_{\beta, \mathrm{p}-\mathrm{t}}}{\left(\omega_{\mathrm{p}} / \omega_{\beta, \mathrm{p}-\mathrm{t}}-1\right) \omega_{\beta, \mathrm{p}-\mathrm{t}}} .
\end{aligned}
$$

Substituting (23) into (25) yields

$$
\frac{\Phi_{\alpha}}{\Phi_{\beta}}=\frac{\omega_{\alpha, \mathrm{p}-\mathrm{t}}}{\omega_{\beta, \mathrm{p}-\mathrm{t}}} \frac{\lambda_{\alpha, \mathrm{p}-\mathrm{t}}^{o / 2}-1}{1-\lambda_{\alpha, \mathrm{p}-\mathrm{t}}} \frac{1-\lambda_{\beta, \mathrm{p}-\mathrm{t}}}{\lambda_{\beta, \mathrm{p}-\mathrm{t}}^{s / 2}-1} .
$$

When scaling factors change within the limit range, that is, $\lambda_{\alpha \mathrm{t}} \rightarrow 1, \lambda_{\beta \mathrm{t}} \rightarrow 1$, there are

$$
\begin{aligned}
\lim _{\lambda_{\alpha, \mathrm{p}-\mathrm{t}} \rightarrow 1, \lambda_{\beta, \mathrm{p}-\mathrm{t}} \rightarrow 1} \frac{\omega_{\alpha, \mathrm{p}-\mathrm{t}}}{\omega_{\beta, \mathrm{p}-\mathrm{t}}} & =1, \\
\lim _{\lambda_{\alpha, \mathrm{p}-\mathrm{t}} \rightarrow 1} \frac{\lambda_{\alpha, \mathrm{p}-\mathrm{t}}^{o / 2}-1}{1-\lambda_{\alpha, \mathrm{p}-\mathrm{t}}} & =-\frac{o}{2}, \\
\lim _{\lambda_{\beta, \mathrm{p}-\mathrm{t}} \rightarrow 1} \frac{1-\lambda_{\beta, \mathrm{p}-\mathrm{t}}}{\lambda_{\beta, \mathrm{p}-\mathrm{t}} s / 2}-1 & =-\frac{2}{s} .
\end{aligned}
$$

So (26) can be denoted as

$$
\frac{\Phi_{\alpha}}{\Phi_{\beta}}=\frac{\omega_{\alpha \mathrm{t}}}{\omega_{\beta \mathrm{t}}}\left(-\frac{o}{2}\right)\left(-\frac{2}{s}\right)=\frac{o}{s} .
$$

When scaling factors change in the little range, this yields

$$
\frac{\Phi_{\alpha}}{\Phi_{\beta}} \approx \frac{o}{s}
$$

In the same way, if the dynamic characteristics of thin walled structures are affected by many parameters, (22) can be obtained according to the recursive relation. Consequently, the additional principle is proved. follows:

Therefore, the basic principles can be summarized as

(1) In distorted scaling laws, if the scaling factor $\lambda_{j}$ is explicit and presented in the governing equation, the index of scaling factor $\lambda_{j}$ can be directly determined.

(2) When sensitivity's absolute values satisfy $\left|\Phi_{1}\right|>$ $\left|\Phi_{2}\right|>\cdots>\left|\Phi_{n}\right|$, the index relation of the scaling factor $\lambda_{n}^{k_{n}}$ is $\left|k_{1}\right|>\left|k_{2}\right|>\cdots>\left|k_{n}\right|$ and $k_{1}: k_{2}: \cdots$ : $k_{n} \approx \Phi_{1}: \Phi_{2}: \cdots: \Phi_{n}$ in the distorted scaling law.

(3) If the sensitivity $\Phi_{j \mathrm{p}}>0, \lambda_{j}$ is positively proportional to $\lambda_{\omega}$ in the distorted scaling law; conversely, $\lambda_{j}$ is inversely proportional to $\lambda_{\omega}$ if the sensitivity $\Phi_{j \mathrm{p}}<0$.

Finally, the procedure of similitude design method of typical thin walled structures is given out.

Step 1. Deducing complete scaling law of typical thin walled structures based on the governing equation.

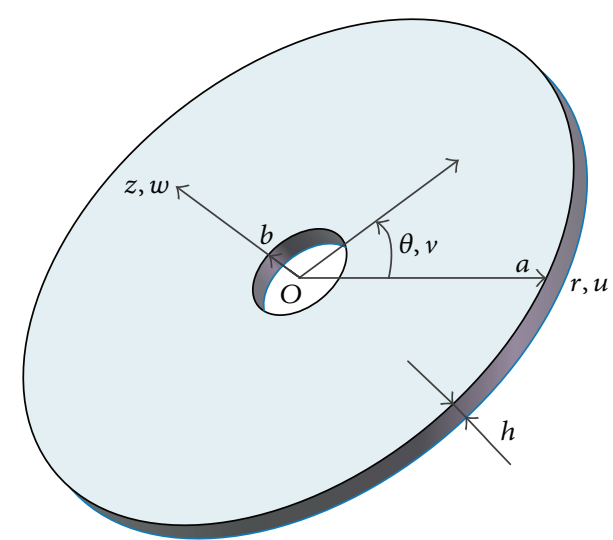

FIGURE 2: The thin walled annular plate.

Step 2. Assuming distorted scaling laws according to the governing equation of typical thin walled structures.

Step 3. Analyzing the sensitivity of the natural frequency with respect to structural parameters.

Step 4. According to the principles for dynamic scaling laws, determining the distorted scaling law of typical thin walled structures.

\section{Numerical Validation}

Take the thin walled annular plate as an example; geometrically complete scaling law of a thin walled annular plate is firstly established. According to the above three principles, the distorted scaling law of the first order's frequency is determined based on the sensitivity analysis. Finally, the determining method of the distorted scaling law is validated by the numerical analysis.

The structure of a thin walled annular plate is shown in Figure 2. $a$ is outer radius, $b$ is inner radius, and $h$ is thickness. The coordinate system $\mathrm{Or} \theta z$ is established, and $O$ is the center point of the end surfaces, $r$ is radial displacement, $\theta$ is deflection angle, and $z$ is axial displacement. Displacement $u$ is along direction $r$, displacement $v$ is along direction $\theta$, and displacement $w$ is along direction $z$.

According to (8), the governing equation of thin walled annular plates can be written as

$$
\begin{aligned}
L_{w-40} & \frac{\partial^{4} W}{\partial r^{4}}+L_{w-42} \frac{\partial^{4} W}{\partial r^{2} \partial \theta^{2}}+L_{w-44} \frac{\partial^{4} W}{\partial \theta^{4}} \\
+ & L_{w-30} \frac{\partial^{3} W}{\partial r^{3}}+L_{w-31} \frac{\partial^{3} W}{\partial r \partial \theta^{2}}+L_{w-20} \frac{\partial^{2} W}{\partial r^{2}} \\
+L_{w} W & =0,
\end{aligned}
$$

where $L_{w-40}=1, L_{w-42}=2 / r^{2}, L_{w-44}=1 / r^{4}, L_{w-30}=2 / r$, $L_{w-31}=2 / r^{3}, L_{w-20}=1 / r^{2}$, and $L_{w}=12 \rho h \omega^{2}\left(1-\mu^{2}\right) / E h^{2}$.

The geometric sizes and material parameters of the thin walled annular plate are listed in Table 1; the boundary condition is clamped on the inner radius edge and free on 
TABLE 1: The geometric sizes and material parameters.

\begin{tabular}{lccccc}
\hline $\begin{array}{l}\text { Outer radius } \\
a / \mathrm{mm}\end{array}$ & $\begin{array}{c}\text { Inner radius } \\
b / \mathrm{mm}\end{array}$ & $\begin{array}{c}\text { Thickness } \\
h / \mathrm{mm}\end{array}$ & $\begin{array}{c}\text { Young's modulus } \\
E / \mathrm{GPa}\end{array}$ & $\begin{array}{c}\text { Density } \\
\rho / \mathrm{kg} \cdot \mathrm{m}^{-3}\end{array}$ & $\begin{array}{c}\text { Poisson's ratio } \\
\mu\end{array}$ \\
\hline 100 & 12 & 6 & 210 & 8000 & 0.3 \\
\hline
\end{tabular}

TABLE 2: The dynamic characteristics of the prototype.

\begin{tabular}{lcccc}
\hline Order $i$ & 1 & 2 & 3 & 4 \\
\hline Natural frequency $\omega / \mathrm{Hz}$ & 541.90 & 647.94 & 836.11 & 1819.9 \\
Vibration modes & $m=2, n=1$ & $m=1, n=1$ & $m=4, n=1$ & $m=6, n=1$
\end{tabular}

TABLE 3: Natural frequencies of the 1st mode $(2,1)$ as a result of the distorted outer radius.

\begin{tabular}{lccc}
\hline & $\begin{array}{c}\text { Outer radius } \\
a / \mathrm{mm}\end{array}$ & $\begin{array}{c}\text { Scaling factor of } \\
\text { outer radius } \lambda_{a}\end{array}$ & $\begin{array}{c}\text { Natural frequencies } \\
\omega_{\mathrm{m}} / \mathrm{Hz}\end{array}$ \\
\hline Model 1 & 96 & 0.96 & 597.47 \\
Model 2 & 98 & 0.98 & 568.69 \\
Model 3 & 102 & 1.02 & 516.92 \\
Model 4 & 104 & 1.04 & 493.59 \\
\hline
\end{tabular}

the outer radius boundary. For vibration experiments, natural frequencies should be proportional between the distorted model and prototype under the condition of the same vibration modes. By using ANSYS, 20-node element SOLID186 is selected to build the thin walled annular plate. Then, analyze its free vibration characteristics, and the previous five orders' natural frequencies and vibration modes are listed in Table 2. $m$ is circumferential half wave number and $n$ is radial half wave number.

4.1. Geometrically Complete Scaling Law. According to the governing equation (30), it yields

$$
\frac{\lambda_{W}}{\lambda_{r}^{4}}=\frac{\lambda_{\rho} \lambda_{\omega}^{2}}{\lambda_{E} \lambda_{h}^{2}} \lambda_{W}
$$

Under the condition of geometrically complete similitude, scaling factors can be expressed as

$$
\lambda_{a}=\lambda_{b}=\lambda_{r}=\lambda_{h}=\lambda, \quad \lambda_{\mu}=1 .
$$

Therefore, geometrically complete scaling law of the thin walled annular plates is

$$
\lambda_{\omega}=\frac{1}{\lambda} \sqrt{\frac{\lambda_{E}}{\lambda_{\rho}}}
$$

4.2. Distorted Scaling Law. In general, scaling factors $\lambda_{a}$ and $\lambda_{b}$ are independent in distorted scaling laws [29]. $\lambda_{r}^{4}=\lambda_{a}^{-o} \lambda_{b}^{-s}$ is considered in (31). In addition, from (31), $o+s=-4$

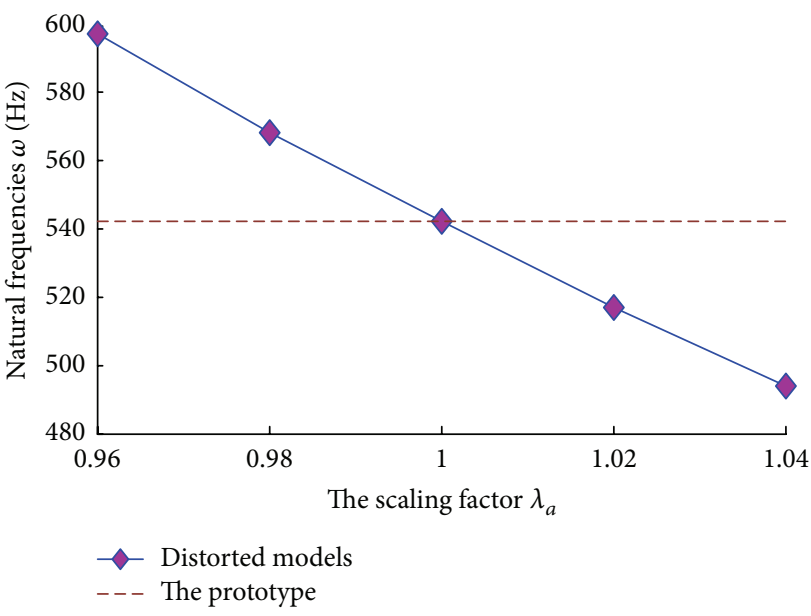

FIGURE 3: The fitted curve of outer radius distorted models.

should be subjected to the distorted scaling law. Therefore, the distorted scaling law of thin walled annular plates can be written as

$$
\begin{aligned}
\lambda_{\omega} & =\lambda_{h} \sqrt{\frac{\lambda_{E}}{\lambda_{\rho} \lambda_{a}^{-o} \lambda_{b}^{-s}}}=\lambda_{h} \sqrt{\frac{\lambda_{E} \lambda_{a}^{o} \lambda_{b}^{s}}{\lambda_{\rho}}}, \\
o+s & =-4 .
\end{aligned}
$$

From (34), the indexes of the scaling factors $\lambda_{E}, \lambda_{\rho}$, and $\lambda_{h}$ have been determined by the governing equation. However, the indexes $o$ and $s$ need to be determined by the sensitivity analysis.

In order to obtain the sensitivity of the first order's natural frequency with respect to the scaling factor $\lambda_{a}$, other sizes and material parameters remain unchanged and the first order's natural frequencies of distorted models are listed in Table 3 by ANSYS simulation.

In Figure 3, the curve is fitted by natural frequencies and the quadratic equation is

$$
\omega_{1 a}=2270 \lambda_{a}^{2}-5837 \lambda_{a}+4109
$$


TABLE 4: Parameters of the distorted model.

\begin{tabular}{lccccc}
\hline $\begin{array}{l}\text { Outer radius } \\
a / \mathrm{mm}\end{array}$ & $\begin{array}{c}\text { Inner radius } \\
b / \mathrm{mm}\end{array}$ & $\begin{array}{c}\text { Thickness } \\
h / \mathrm{mm}\end{array}$ & $\begin{array}{c}\text { Young's modulus } \\
E / \mathrm{GPa}\end{array}$ & $\begin{array}{c}\text { Density } \\
\rho / \mathrm{kg} \cdot \mathrm{m}^{-3}\end{array}$ & $\begin{array}{c}\text { Poisson's ratio } \\
\mu\end{array}$ \\
\hline 130 & 12 & 8 & 72 & 2700 & 0.3 \\
\hline
\end{tabular}

TABLE 5: The distorted scaling laws.

\begin{tabular}{|c|c|c|c|c|c|c|}
\hline $\begin{array}{l}\text { Order } \\
i\end{array}$ & $\begin{array}{c}\text { Sensitivity } \\
\Phi_{a}\end{array}$ & $\begin{array}{c}\text { Sensitivity } \\
\Phi_{b}\end{array}$ & Distorted scaling laws & $\begin{array}{c}\text { Frequencies of model } \\
\omega_{\mathrm{m}} / \mathrm{Hz}\end{array}$ & $\begin{array}{c}\text { Predicted frequencies } \\
\omega_{\mathrm{pr}} / \mathrm{Hz}\end{array}$ & $\begin{array}{c}\text { Error } \\
\eta / \% \\
\end{array}$ \\
\hline 1 & -1297 & 232.3 & $\lambda_{\omega}=\lambda_{h} \sqrt{\frac{\lambda_{E} \lambda_{b}^{0.61}}{\lambda_{\rho} \lambda_{a}^{4.61}}}$ & 387.56 & 527.33 & 2.69 \\
\hline 2 & -1437 & 144.9 & $\lambda_{\omega}=\lambda_{h} \sqrt{\frac{\lambda_{E} \lambda_{b}^{0.36}}{\lambda_{\rho} \lambda_{a}^{4.36}}}$ & 490.27 & 645.64 & 0.35 \\
\hline 3 & -1765 & 109.1 & $\lambda_{\omega}=\lambda_{h} \sqrt{\frac{\lambda_{E} \lambda_{b}^{0.23}}{\lambda_{\rho} \lambda_{a}^{4.23}}}$ & 647.17 & 837.91 & 0.22 \\
\hline 4 & -3621 & 10 & $\lambda_{\omega}=\lambda_{h} \sqrt{\frac{\lambda_{E} \lambda_{b}^{0.01}}{\lambda_{\rho} \lambda_{x}^{4.01}}}$ & 1445.2 & 1818.1 & 0.10 \\
\hline 5 & -6247 & 5 & $\lambda_{\omega}=\lambda_{h} \sqrt{\frac{\lambda_{E}}{\lambda_{\rho} \lambda_{a}^{4}}}$ & 2517.5 & 3162.9 & 0.16 \\
\hline
\end{tabular}

TABLE 6: The errors between the distorted models and prototype.

\begin{tabular}{lccccccc}
\hline Model & $\lambda_{a}$ & $\lambda_{b}$ & $\lambda_{h}$ & $\Gamma$ & $\Psi$ & $\begin{array}{c}\text { Frequencies of model } \\
\omega_{\mathrm{m}} / \mathrm{Hz}\end{array}$ & $\begin{array}{c}\text { Predicted 1st frequencies } \\
\omega_{\mathrm{pr}} / \mathrm{Hz}\end{array}$ \\
\hline M1 & 1 & 0.6 & 0.6 & 1.67 & 1.67 & 274.72 & 535.06 \\
M2 & 1 & 0.8 & 0.8 & 1.25 & 1.25 & 400.15 & 535.41 \\
M3 & 1 & 1.2 & 1.2 & 0.83 & 0.83 & 700.91 & 552.49 \\
M4 & 1 & 1.4 & 1.4 & 0.71 & 0.71 & 878.43 & 566.25 \\
M5 & 1 & 1.2 & 0.6 & 0.71 & 1.67 & 357.47 & 563.55 \\
\hline
\end{tabular}

Furthermore, adopting the adjusted square $\bar{R}^{2}$ to verify the efficiency of the fitted curve, the adjusted square $\bar{R}^{2}$ can be denoted as

$$
\begin{array}{r}
\bar{R}^{2}=1-\left[\frac{n^{\prime}-1}{n^{\prime}-(k+1)}\right]\left(1-\frac{\sum\left(\widehat{Y}_{i}-\bar{Y}\right)^{2}}{\sum\left(Y_{i}-\bar{Y}\right)^{2}}\right) \\
\left(i=1,2, \ldots, n^{\prime}\right),
\end{array}
$$

where $n^{\prime}$ is fitted point numbers; $k$ is the order of fitted polynomial; $\hat{Y}$ is the fitted value; $\bar{Y}$ is the average value; $Y$ is the true value.

By comparing and analyzing, the fitted curve is thought to be effective and suitable if $\bar{R}^{2}>0.99$ in this paper.

The adjusted square of the fitted curve is $\bar{R}_{a}^{2}=0.999$. So the fitted curve of the first order's natural frequency is effective.

The sensitivity of the natural frequency with respect to the scaling factor $\lambda_{a}$ is

$$
\Phi_{1 a}=\left.\frac{\mathrm{d} \omega_{1 a}}{\mathrm{~d} \lambda_{a}}\right|_{\lambda_{a}=1}=4540 \lambda_{a}-5837=-1297
$$

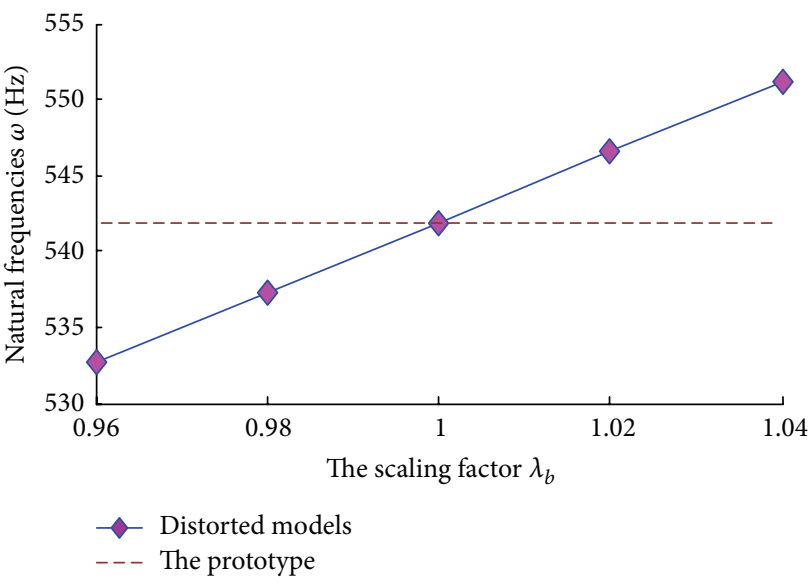

FIGURE 4: The fitted curve of inner radius distorted models.

In the same way, the fitted curve of the first order's natural frequencies of inner radius distorted models is shown in Figure 4.

The fitted equation of the first order's natural frequency is

$$
\omega_{1 b}=5.4 \lambda_{b}^{2}+221.5 \lambda_{b}+315
$$


TABLE 7: Parameters of experimental plates.

\begin{tabular}{lccccrr}
\hline & $\begin{array}{c}\text { Outer radius } \\
a / \mathrm{mm}\end{array}$ & $\begin{array}{c}\text { Inner radius } \\
b / \mathrm{mm}\end{array}$ & $\begin{array}{c}\text { Thickness } \\
h / \mathrm{mm}\end{array}$ & $\begin{array}{c}\text { Young's modulus } \\
E / \mathrm{GPa}\end{array}$ & $\begin{array}{c}\text { Poisson's ratio } \\
\mu\end{array}$ & $\begin{array}{c}\text { Density } \\
\rho / \mathrm{kg} \cdot \mathrm{m}^{-3}\end{array}$ \\
\hline Prototype & 100 & 12 & 6 & 210 & 0.3 & 8000 \\
Model & 130 & 12 & 8 & 72 & 0.3 & 2700 \\
\hline
\end{tabular}

TABLE 8: The comparison of experimental and predicted results.

\begin{tabular}{|c|c|c|c|c|c|c|c|}
\hline $\begin{array}{l}\text { Orders } \\
i\end{array}$ & $\begin{array}{l}\text { Model } \\
\omega_{\mathrm{m}} / \mathrm{Hz} \\
\end{array}$ & Vibration modes & $\begin{array}{c}\text { Prototype } \\
\omega_{\mathrm{p}} / \mathrm{Hz}\end{array}$ & Vibration modes & Modes & $\begin{array}{c}\text { Predicted frequencies } \\
\omega_{\mathrm{pr}} / \mathrm{Hz}\end{array}$ & $\begin{array}{c}\text { Errors } \\
\eta / \% \\
\end{array}$ \\
\hline 1 & 639.34 & & 862.31 & & $m=4, n=1$ & 827.77 & 4.01 \\
\hline 2 & 1431.5 & & 1842.5 & & $m=6, n=1$ & 1798.5 & 2.39 \\
\hline 3 & 2512.8 & & 3203.6 & & $m=8, n=1$ & 3157.1 & 1.45 \\
\hline
\end{tabular}

In Figure 4, the adjusted square of the fitted curve is $\bar{R}_{b}^{2}=$ 0.999 , so the fitted curve is thought to be effective.

The sensitivity of the first order's natural frequency with respect to the scaling factor $\lambda_{b}$ is

$$
\Phi_{1 b}=\left.\frac{\mathrm{d} \omega_{1 b}}{\mathrm{~d} \lambda_{b}}\right|_{\lambda_{b}=1}=10.8 \lambda_{b}+221.5=232.3 .
$$

As a result of the sensitivity analysis,

$$
\Phi_{a}: \Phi_{b}=\left.\frac{\mathrm{d} \omega_{1 a}}{\mathrm{~d} \lambda_{a}}\right|_{\lambda_{a}=1}:\left.\frac{\mathrm{d} \omega_{1 b}}{\mathrm{~d} \lambda_{b}}\right|_{\lambda_{b}=1}=-1297: 232.3 .
$$

According to Principle 2, this yields

$$
o: s=-1297: 232.3 \text {. }
$$

From (34), $o+s=-4$ is satisfied.

Solving simultaneous equations yields

$$
\begin{aligned}
& o=-4.61, \\
& s=0.61 .
\end{aligned}
$$

Therefore, the distorted scaling law of the first order's natural frequency of thin walled annular plates can be written as

$$
\lambda_{1 \omega}=\lambda_{h} \sqrt{\frac{\lambda_{E} \lambda_{b}^{0.61}}{\lambda_{\rho} \lambda_{a}^{4.61}}}
$$

4.3. Numerical Validation. In the same way, the previous five orders' distorted scaling laws are determined and the effectiveness of distorted similitude models is validated. Finally, the procedure of determining the accurate distorted scaling laws is presented.

The geometric sizes and material parameters of the distorted model are listed in Table 4.
Similarly, the sensitivity of the natural frequencies with the scaling factors $\lambda_{a}$ and $\lambda_{b}$ can be obtained. By using the sensitivity value, the previous five orders' distorted scaling laws of thin walled annular plates are shown in Table 5.

The error between the natural frequency of the prototype and the predictive natural frequency can be denoted as

$$
\eta=\frac{\left|\omega_{\mathrm{p}}-\omega_{\mathrm{pr}}\right|}{\omega_{\mathrm{p}}} \times 100 \%
$$

Table 5 depicts that errors between the natural frequencies of the prototype and predicted values are less than $5 \%$, and their modes are the same. Therefore, the previous five orders' distorted scaling laws can accurately predict the dynamic characteristics of a prototype.

$\Gamma$ is defined as $\Gamma=\lambda_{a} / \lambda_{b}$, and $\Psi$ is defined as $\Psi=\lambda_{a} / \lambda_{h}$. Different distorted models are selected and the scaling factors are shown in Table 6. Taking the 1st frequency as an example, the 1st frequencies of distorted models are obtained by using ANSYS, and the error can be calculated by (44). Furthermore, the predicted frequencies and errors are shown in Table 6.

\section{Experiments Validation}

In the experiment, test setup of the experimental plate is shown in Figure 5 and parameters of the experimental plates are listed in Table 7.

In addition, Table 8 depicts the vibration modes of the prototype and model. Errors between the predicted values and experimental values of the prototype are listed in Table 8.

From Table 8, errors between the previous five orders' natural frequencies of experimental prototype and predicted values are less than $5 \%$, and vibration modes of experimental prototype and model are the same.

The experimental errors are higher than the numerical errors; the reasons are analyzed as follows: 


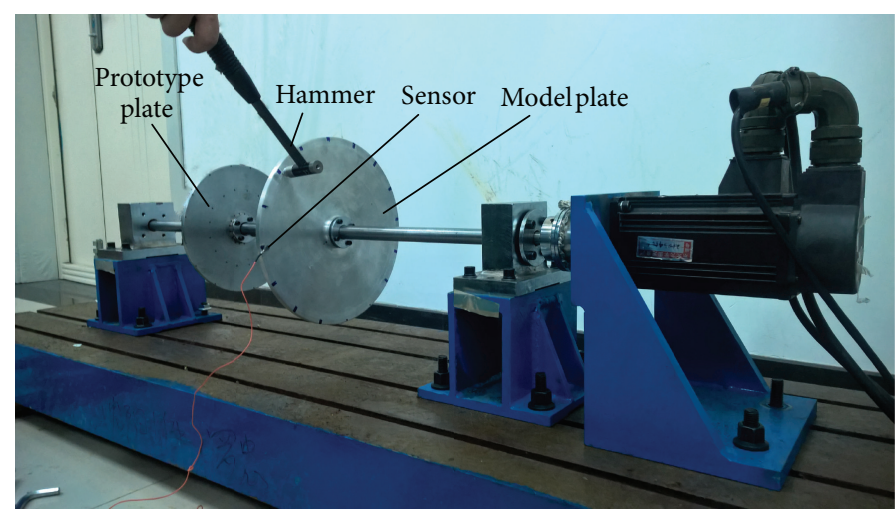

FIGURE 5: Test setup of the experimental plate.

(1) The machining of the thin walled annular plates, such as the dimensional error and deviation of material parameters.

(2) The measurement accuracy of a test system, for example, the precision of the sensor.

(3) The random error of the test procession.

\section{Conclusions}

In this paper, in order to investigate the similitude design method of typical thin walled structures, the governing equation of typical thin walled structures is firstly established and the geometrically complete scaling law is deduced. In order to determine accurate distorted scaling laws of thin walled structures, three principles are proposed and theoretically proved by combining the governing equation and sensitivity analysis. Taking the thin walled annular plate as an example, geometrically complete and distorted scaling laws are obtained based on the three principles. Finally, the design method of similitude models of typical thin walled structures is validated via experiments, and detailed conclusions are listed as follows:

(1) The governing equation of typical thin walled structures is unified in (8).

(2) By employing the governing equation, geometrically complete scaling law of typical thin walled structures is obtained.

(3) In order to determine the accurate distorted scaling law of thin walled structures, three principles are proposed and theoretically proved by combining the governing equation and sensitivity analysis.

(4) Taking thin walled annular plate as an example, the design method of similitude models of thin walled structures is validated by numerical simulation and experiments.

There are also some restrictions about the similitude design method; one of the limitations is that a numerical model of the thin walled structure is always necessary to compute the sensitivity.

\section{Conflict of Interests}

The authors declare that there is no conflict of interests regarding the publication of this paper.

\section{Acknowledgments}

The authors gratefully acknowledge the financial support from the Fundamental Research Funds for the Central Universities of China (Grant nos. N130503001 and N140301001), the Key Laboratory for Precision \& Nontraditional Machining of Ministry of Education, Dalian University of Technology (Grant no. JMTZ201602), and the National Program on Key Basic Research Project (Grant no. 2012CB026000).

\section{References}

[1] V. Ungbhakorn and P. Singhatanadgid, "Similitude invariants and scaling laws for buckling experiments on antisymmetrically laminated plates subjected to biaxial loading," Composite Structures, vol. 59, no. 4, pp. 455-465, 2003.

[2] Z. Y. Qin, Q. K. Han, and F. L. Chu, "Analytical model of bolted disk-drum joints and its application to dynamic analysis of jointed rotor," Proceedings of the Institution of Mechanical Engineers Part C: Journal of Mechanical Engineering Science, vol. 228, no. 4, pp. 646-663, 2014.

[3] Y. Narita, "Natural frequencies of free, orthotropic elliptical plate," Journal of Sound and Vibration, vol. 100, no. 1, pp. 83-89, 1985.

[4] X. Wang, G. Striz, and W. Bert, "Free vibration analysis of annular plates by the DQ method," Journal of Sound and Vibration, vol. 164, no. 1, pp. 173-175, 1993.

[5] T. Irie, G. Yamada, and Y. Kaneko, "Free vibration of a conical shell with variable thickness," Journal of Sound and Vibration, vol. 82, no. 1, pp. 83-94, 1982.

[6] S. O. Papkov and J. R. Banerjee, "A new method for free vibration and buckling analysis of rectangular orthotropic plates," Journal of Sound and Vibration, vol. 339, pp. 342-358, 2015.

[7] B. Krayterman and G. M. Sabnis, "Similitude theory: plates and shells analysis," Journal of Engineering Mechanics, vol. 110, no. 9, pp. 1247-1263, 1984. 
[8] Y. Qian and S. R. Swanson, "Experimental measurement of impact response in carbon/epoxy plates," AIAA Journal, vol. 28, no. 6, pp. 1069-1074, 1990.

[9] G. J. Simitses and J. Rezaeepazhand, "Structural similitude for laminated structures," Composites Engineering, vol. 3, no. 7-8, pp. 751-765, 1993.

[10] J. Rezaeepazhand and G. J. Simitses, "Design of scaled down models for predicting shell vibration response," Journal of Sound and Vibration, vol. 195, no. 2, pp. 301-311, 1996.

[11] J. Rezaeepazhand and G. J. Simitses, "Structural similitude for vibration response of laminated cylindrical shells with double curvature," Composites Part B: Engineering, vol. 28, no. 3, pp. 195-200, 1997.

[12] J.-J. Wu, M. P. Cartmell, and A. R. Whittaker, "Prediction of the vibration characteristics of a full-size structure from those of a scale model," Computers and Structures, vol. 80, no. 18-19, pp. 1461-1472, 2002.

[13] J.-J. Wu, "The complete-similitude scale models for predicting the vibration characteristics of the elastically restrained flat plates subjected to dynamic loads," Journal of Sound and Vibration, vol. 268, no. 5, pp. 1041-1053, 2003.

[14] P. Singhatanadgid and V. Ungbhakorn, "Scaling laws for vibration response of anti-symmetrically laminated plates," Structural Engineering and Mechanics, vol. 14, no. 3, pp. 345-364, 2002.

[15] P. Singhatanadgid and V. Ungbhakorn, "Scaling laws for buckling of polar orthotropic annular plates subjected to compressive and torsional loading," Thin-Walled Structures, vol. 43, no. 7, pp. 1115-1129, 2005.

[16] V. Ungbhakorn and N. Wattanasakulpong, "Structural similitude and scaling laws of anti-symmetric cross-ply laminated cylindrical shells for buckling and vibration experiments," International Journal of Structural Stability and Dynamics, vol. 7, no. 4, pp. 609-627, 2007.

[17] R. E. Oshiro and M. Alves, "Scaling of structures subject to impact loads when using a power law constitutive equation," International Journal of Solids and Structures, vol. 46, no. 18-19, pp. 3412-3421, 2009.

[18] R. E. Oshiro and M. Alves, "Scaling of cylindrical shells under axial impact," International Journal of Impact Engineering, vol. 34, no. 1, pp. 89-103, 2007.

[19] R. E. Oshiro and M. Alves, "Predicting the behaviour of structures under impact loads using geometrically distorted scaled models," Journal of the Mechanics and Physics of Solids, vol. 60, no. 7, pp. 1330-1349, 2012.

[20] A. A. Yazdi, "Study nonlinear vibration of cross-ply laminated plates using scale models," Polymer Composites, vol. 35, no. 4, pp. 752-758, 2014.

[21] S. De Rosa, F. Franco, and V. Meruane, "Similitudes for the structural response of flexural plates," Proceedings of the Institution of Mechanical Engineers Part C: Journal of Mechanical Engineering Science, 2015.

[22] S. De Rosa, F. Franco, and T. Polito, "Structural similitudes for the dynamic response of plates and assemblies of plates," Mechanical Systems and Signal Processing, vol. 25, no. 3, pp. 969980, 2011.

[23] Z. Luo, Y. P. Zhu, X. B. Chen, and X. Y. Zhao, "Determination method of the structure size intervals of dynamic distorted model of elastic cantilever thin plate," Advances in Mechanical Engineering, vol. 6, Article ID 791047, 2014.
[24] Z. Luo, X. Y. Zhao, Y. P. Zhu, and J. Z. Li, "Determination method of the structure size interval of dynamic similar models for predicting vibration characteristics of the isotropic sandwich plates," Journal of Vibroengineering, vol. 16, no. 2, pp. 608-622, 2014.

[25] W. Soedel, Vibrations of Shells and Plates, CRC Press, London, UK, 2004.

[26] Z. Luo, Y. Zhu, X. Zhao, and D. Wang, "Determination method of dynamic distorted scaling laws and applicable structure size intervals of a rotating thin-wall short cylindrical shell," Proceedings of the Institution of Mechanical Engineers, Part C: Journal of Mechanical Engineering Science, vol. 229, no. 5, pp. 806-817, 2015.

[27] I.-W. Lee and G.-H. Jung, "An efficient algebraic method for the computation of natural frequency and mode shape sensitivities. Part I. Distinct natural frequencies," Computers and Structures, vol. 62, no. 3, pp. 429-435, 1997.

[28] I.-W. Lee and G.-H. Jung, "An efficient algebraic method for the computation of natural frequency and mode shape sensitivities-Part II. Multiple natural frequencies," Computers and Structures, vol. 62, no. 3, pp. 437-443, 1997.

[29] H. Bisadi, M. Es'haghi, H. Rokni, and M. Ilkhani, "Benchmark solution for transverse vibration of annular Reddy plates," International Journal of Mechanical Sciences, vol. 56, no. 1, pp. 35-49, 2012. 


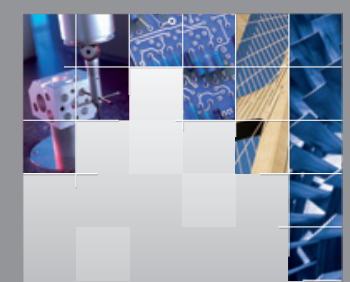

\section{Enfincering}
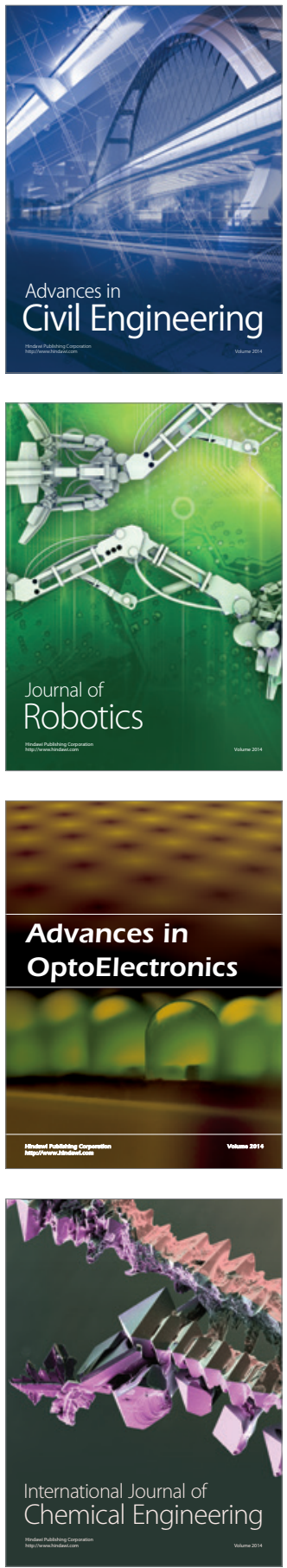

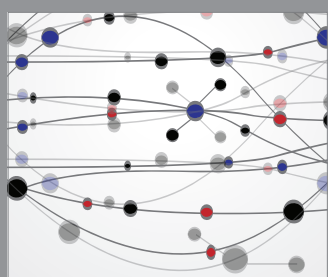

The Scientific World Journal

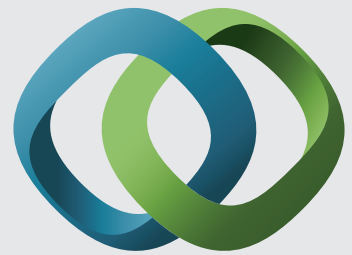

\section{Hindawi}

Submit your manuscripts at

http://www.hindawi.com
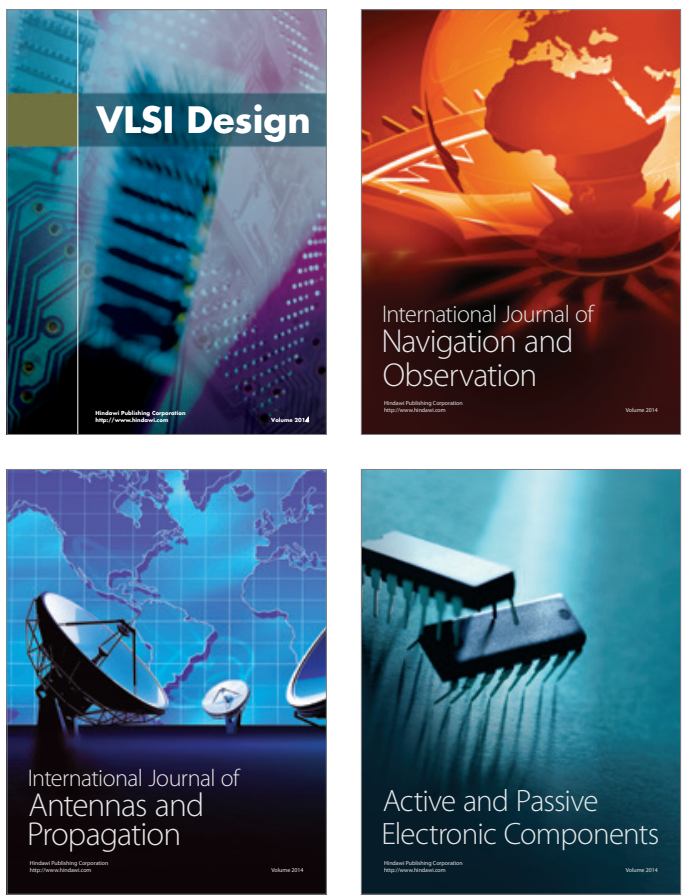
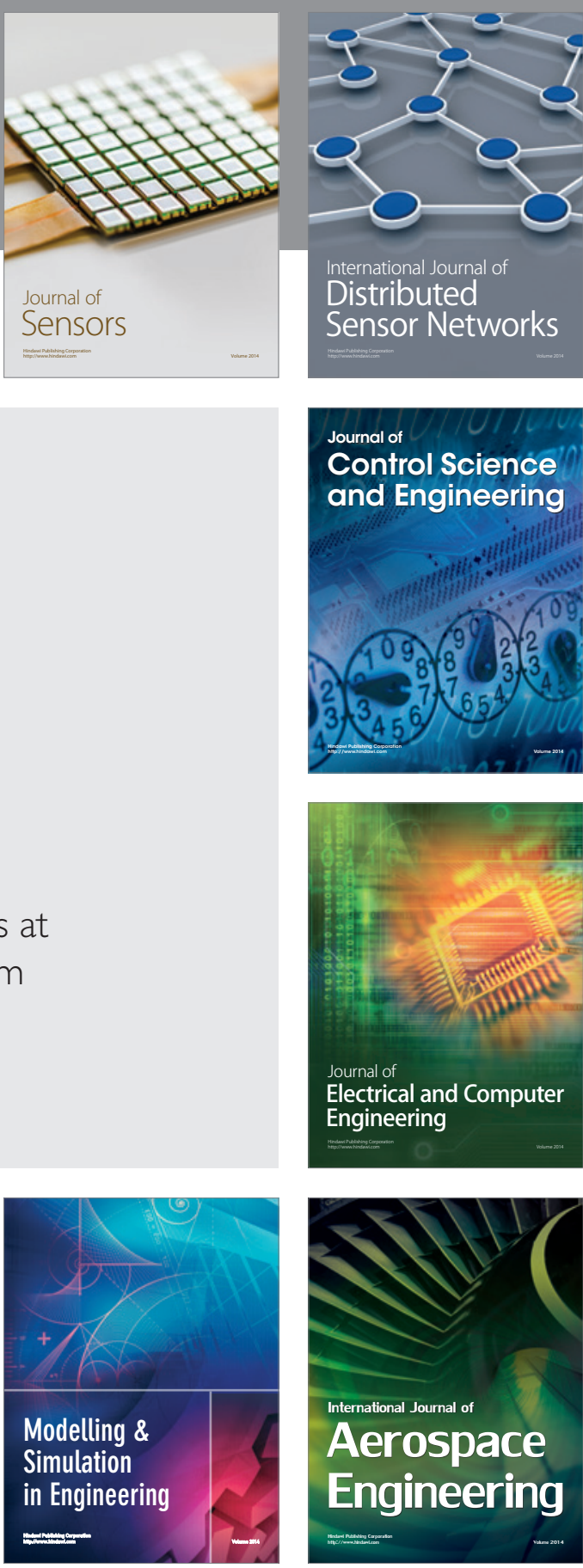

International Journal of

Distributed

Sensor Networks

Journal of

Control Science

and Engineering
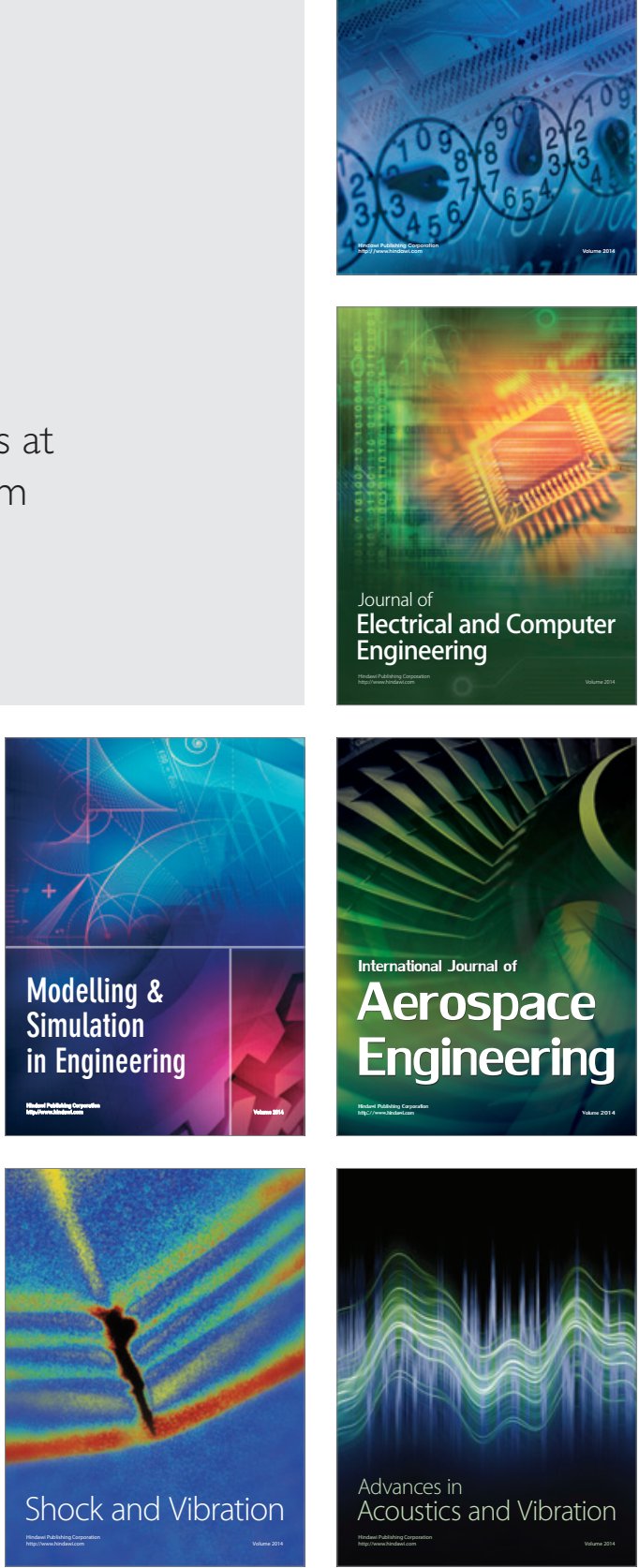